The work being reviewed resembles several others in this and related fields, coming from the United States, in that attractively constructed examples are described and worked out in the text, under headings like "The Tomato Soup Company's Problem". Reading the latter example we find data about prior probabilities, costs of various actions, information available about observable random variables, and so on, motivated and supported by a lively story about a production manager who has to decide how many tons of tomatoes to buy. The problem is worked out, with the accompanying story bringing in further data as required, to the conclusion that he should contract for about 375 acres of tomatoes.

Most of the characteristics of the book are exemplified in "The Tomato Soup Company's Problem". The decision process described is Bayesian and the production manager in the story firmly adopts a normal prior distribution for the yield of tomatoes, having a mean of 60 tons/acre and a standard deviation of 4 tons. The examples are thoroughly numerical, though the figures have surely been organized so that none of the calculations get messy. The text accompanying the calculations is descriptive and colourful, which accounts to some extent for the $\mathbf{5 8 0}$ pages to which the book extends. The length of the book, however, is also due to the large amount of material in it. A reader who has started with finite event sets in the first chapter will be using posterior distributions for jointly continuous distributions by the time he reaches the "Tomato Soup" problem in the eighth chapter, having been taken through a mass of technical material on the way. In connexion with continuous distributions we note that sections using the calculus are specially marked and the book is so designed that a great deal can be read without even this tool. Mathematical topies like the gamma function and $\lim _{p \rightarrow 0}(1-p)^{1 / p}$ are outlined, although the level is illustrated by the fact that the latter is shown numerically to be "approaching a number whose value is $0 \cdot 367$ ", followed by a remark about proof, and a reference.

The contents of the book are divided fairly evenly between the two subjects in the title. The first, fifth, sixth and seventh chapters provide a fairly standard treatment of probability theory, from finite event sets up to a statement, without proof, of the central limit theorem. The second chapter introduces the idea of utility, and decision problems are dealt with in the third and fourth chapters. In the eighth chapter the two threads of the book are brought together to deal with "problems which have the characteristic that in determining the probabilities of the states of nature we make use of historical data or personal feelings and, in addition, make use of the results of a random experiment". In the ninth chapter the classical techniques of hypothesis testing and estimation are introduced and their relation with the decision-theoretic methods in the book is outlined. The tenth chapter treats the Poisson process and in the final chapter sequential decision problems are introduced.

This book is stimulating in many ways and in the United States it will clearly be a saleable addition to Holden-Day's series on "Industrial Engineering and Management Science". It is harder to see a place for it in a British university syllabus. It might be useful in a degree course in mathematics and statistics, but most students in such a course would not need the lengthy numerical illustrations and the partial avoidance of calculus. Postgraduate students in operational research would also, probably, have sufficient background to find the pace of this book slow. Nevertheless, instructors in such courses would find it a useful source and a good book to refer some students to after a tutorial. It could well be just the book for self-instruction by someone who decided to take up the subject on his own.

W. A. O'N. WAUGH

\section{SOURCE BOOK OF SEPARATION}

\section{Chromatography}

By Erich Heftmann. Second edition. (Reinhold Chemistry Textbook Series.) Pp. xli +851 . (New York and London: Reinhold Publishing Corporation, 1967.) $220 s$.

THIs is the second edition of the book published in 1961 and reprinted in 1963 . There has been much revision; obsolete matter has been eliminated while latest developments have been incorporated and several new chapters have been added. The author index has been eliminated, which I think is a mistake, especially because the authors of publications listed at the end of each chapter are not arranged alphabetically. The book is intended as a reference work and also "as a supplementary textbook for graduate school courses in analytical ohemistry, biochemistry and chemical engincering". Whether this applies to students or teachers is not clear, but whatever their standard of living in the United States, very few in this country could afford to buy the book.

This book is a collective work with contributions from more than thirty authors, and is organized in two parts. The first deals with theories and techniques of all the forms of chromatography and includes electrophoresis. The second part contains chapters which cover the applications of all these techniques to particular classes of compound. Much of this part covers the literature to 1965 , which is a praiseworthy achievement, but some sections do not go so far and consequently are already outdated, examples being gas chromatography of sugars and of amino-acids. Some sections of this second part tend to be highly concentrated reviews which make them rather difficult reading, and I feel that more use could have been made of tables which would have made it easier for the reader to obtain information. This has been done with good effect in the chapters on nucleic acids, phenolics, terpenes, non-hydrocarbon and hydrocarbon gases.

The first part of the book generally reaches a high standard. The two chapters on the general theory of chromatography and on the theoretical basis of partition chromatography are particularly valuable contributions. Techniques are covered adequately and the chapters are well written expositions of principles and practical aspects. This part does not suffer from the shortcomings of the second part which perhaps are inevitable, as one cannot expect a single book to cover such a vast subject fully.

Nevertheless, this volume contains a great deal of useful, comprehensive and up to date information and is probably the best general book on chromatography which exists.

R. CONSDEN

\section{ENVIRONMENTAL RADIOACTIVITY}

\section{Radioecological Concentration Processes}

Edited by Bertil Aberg and Frank P. Hungate. (Proceedings of an International Symposium held in Stockholm on April 25-29, 1966.) Pp. xiv + 1040. (Oxford, London and New York: Pergamon Press, Ltd., 1967.) $300 s$. net.

MAN began the spread of artificial contaminants through his environment when he lit his first smoking fire, and from this small beginning he has gone on to pollute air, land and water with a wide variety of toxic substances. Five to ten years ago, the contaminant which aroused greatest public alarm was the radioactive fallout from nuclear weapons exploded in the atmosphere. Undoubtedly, some of this alarm was an expression of general unease at the very existence of the weapons, but it did 